\title{
COVID-19 vaccination clinical trials should consider multiple doses of BCG
}

Bassam M. Ayoub

The Center for Drug Research and Development (CDRD), Pharmaceutical Chemistry Department, Faculty of Pharmacy, The British University in Egypt, El-Sherouk city, Cairo, Egypt.

* Corresponding author, Associate Professor Bassam M. Ayoub, Ph.D.

Pharmaceutical Chemistry Department

The Center for Drug Research and Development (CDRD)

Faculty of Pharmacy, The British University in Egypt

Suez Road, El Sherouk City, Cairo Governorate 11837

Phone: +20226890000; +201225104337

FAX: +20226300010

bassam.ayoub@bue.edu.eg 
Vaccine repositioning is a hot research topic as an alternative to the traditional vaccine approach, which is a costly and time-consuming process due to the availability of previous safety and toxicology data. Multiple-dose BCG vaccine repurposing for COVID-19 will be an uprising breakthrough of vaccine discovery with safer outcomes. BCG induces cross-protection that might not be related to the target disease as innate immune cells, including monocytes and natural killer cells, contribute to this immune protection as known as "trained immunity" [1]. BCG had multifaceted protection against TB, Leprosy \& heterogeneous pathogens [2]. Moreover, it was repositioned as a treatment for type-1 diabetes, many types of cancer \& multiple sclerosis [2]. BCG vaccine accelerates the "resetting" of the immune system [3] or "turn on" immunity mechanism that agrees with its pleiotropic repurposing for many diseases. Multiple-dose BCG vaccine was used for reversing type-1 diabetes \& for treating bladder cancer [4-5]. While intravesical multiple doses of BCG for bladder cancer showed many complications [6-7], intradermal multiple doses of BCG for diabetes showed high safety profile [4].

As recent studies have shown that upon certain vaccinations, human innate immune cells can undergo extensive metabolic and epigenetic reprogramming, which results in enhanced immune responses upon heterologous re-infection, a process termed trained immunity [8]; The author recommends that COVID-19 vaccination clinical trials should consider multiple doses of BCG. After reviewing the recent COVID-19 literature, although some preliminary studies suggested BCG to fight COVID-19 [9-12], they did not consider the use of multiple intradermal BCG vaccination (at least 2 doses, 4 weeks apart [4]) for the prophylaxis of COVID-19 outbreak. I do recommend that diabetic patients should participate in clinical trials to benefit from the 
Letter to the editor

reported BCG anti-hyperglycemic effect [4]. What if safe multiple doses of BCG turned on the immunity and protected people from COVID-19 more efficiently than a single dose?

\section{Conflict of interest}

The author declares that he has no known competing financial interests or personal relationships that could have appeared to influence the multiple dose BCG vaccination suggested in this "letter to the editor".

\section{References}

[1] Covián, C., Fernández-Fierro, A., Retamal-Díaz, A., Díaz, F.E., Vasquez, A.E., Lay, M.K., Riedel, C.A., González, P.A., Bueno, S.M., Kalergis, A.M. BCG-Induced Cross-Protection and Development of Trained Immunity: Implication for Vaccine Design (2019) Frontiers in Immunology, 10, art. no. 2806.

[2] Yamazaki-Nakashimada, M.A., Unzueta, A., Berenise Gámez-González, L., GonzálezSaldaña, N., Sorensen, R.U. BCG: a vaccine with multiple faces (2020) Human Vaccines and Immunotherapeutics, article in press.

[3] Kühtreiber, W.M., Faustman, D.L. BCG Therapy for Type 1 Diabetes: Restoration of Balanced Immunity and Metabolism (2019) Trends in Endocrinology and Metabolism, 30 (2), pp. 80-92.

[4] Kühtreiber, W.M., Tran, L., Kim, T., Dybala, M., Nguyen, B., Plager, S., Huang, D., Janes, S., Defusco, A., Baum, D., Zheng, H., Faustman, D.L. Long-Term reduction in hyperglycemia in advanced type 1 diabetes: The value of induced aerobic glycolysis with BCG vaccinations (2018) npj Vaccines, 3 (1), art. no. 23, . 
[5] Pettenati, C., Ingersoll, M.A. Mechanisms of BCG immunotherapy and its outlook for bladder cancer (2018) Nature Reviews Urology, 15 (10), pp. 615-625.

[6] Waked, R., Choucair, J., Chehata, N., Haddad, E., Saliba, G. Intravesical Bacillus CalmetteGuérin (BCG) treatment's severe complications: A single institution review of incidence, presentation and treatment outcome (2020) Journal of Clinical Tuberculosis and Other Mycobacterial Diseases, 19, art. no. 100149, .

[7] Yong, C., Steinberg, R.L., O'Donnell, M.A. Severe Infectious Complications of Intravesical Bacillus Calmette-Guérin: A Case Series of 10 Patients (2020) Urology, 137, pp. 79-83.

[8] Mourits, V.P., Wijkmans, J.C., Joosten, L.A., Netea, M.G. Trained immunity as a novel therapeutic strategy (2018) Current Opinion in Pharmacology, 41, pp. 52-58.

[9] Can a century-old TB vaccine steel the immune system against the new coronavirus?, https://www.sciencemag.org/news/2020/03/can-century-old-tb-vaccine-steel-immune-systemagainst-new-coronavirus, [accessed 24 March 2020].

[10] Immune boost against the corona virus, https://www.mpg.de/14610776/immune-boostcorona-virus, [accessed 24 March 2020].

[11] This Vaccine Could Save Health Care Workers' Lives Now, https://foreignpolicy.com/2020/03/24/coronavirus-vaccine-health-care-workers-bcg/, , [accessed 24 March 2020].

[12] TB vaccine considered in fight against coronavirus, https://www.brusselstimes.com/allnews/belgium-all-news/101057/tb-vaccine-considered-in-fight-against-coronavirus/, , [accessed 24 March 2020]. 